\title{
An Inference Engine Based on Interval Type-2 Fuzzy BK Subproduct
}

\author{
Chee Kau Lim and Chee Seng Chan \\ Centre of Image and Signal Processing \\ Faculty of Computer Science and Information Technology \\ University of Malaya, 50603 Kuala Lumpur, MALAYSIA \\ Email: limck@siswa.um.edu.my, cs.chan@um.edu.my
}

\begin{abstract}
Recently, Bandler-Kohout (BK) subproduct based reasoning scheme has been a popular choice in various kind of applications. In this paper, we aim to enhance the BK subproduct based reasoning schemes in two aspects: (1) Extend the BK subproduct in term of Interval Type-2 Fuzzy Sets (IT2FS) instead of the ordinary Type-1 Fuzzy Sets (T1FS), and (2) Introduce weight parameter to the reasoning scheme. Firstly, studies have shown that IT2FS have better capability in handling data with uncertainty compare to the ordinary T1FS. Thus, we extend the BK subproduct in terms of IT2FS theory where subsethood measure based on the fuzzy implication operators for the IT2FS has been developed. Secondly, weight parameter associated to each features is introduced to form a weighted inference scheme with the BK subproduct. The introduction of the weight parameter is to aid in distinguishing the influence of different features in the reasoning process. In here, the Linguistic Weighted Average (LWA) is adopted to solve the outputs of this weighted reasoning scheme. Finally, a case study is employed to demonstrate the capability of the proposed approach.
\end{abstract}

\section{INTRODUCTION}

Recent studies [1], [2] have risen the excellency of fuzzy implication based deductive reasoning schemes, such as the Bandler-Kohout (BK) subproduct [3], [4]. These studies showed the BK subproduct based reasoning scheme able to demonstrate and match all the important properties of the much more popular reasoning scheme, namely the Compositional Rule of Inference (CRI) [5]. This has deducted that BK subproduct is competent to form effective and efficient inference engines if appropriate rules are given.

Apart from the rule based reasoning discussed in [1], [2], BK subproduct also well known with its great achievements in case based reasoning. Some successful examples that can be found in the literature include medical expert systems [6], [7], intelligent navigation systems [8], information retrieval [9], land evaluation [10], cognitive sciences [11], scene understanding [12] and etc.

Despite of its successfulness, there are some limitations associated in the current BK subproduct in case based reasoning. First of all, the implementations of BK subproduct in the literature are still based on classical Type-1 Fuzzy Sets (T1FS) theory, which address uncertainty with point-values. Studies such as [13], [14] claimed that T1FS has its limitation in addressing uncertainty with its crisp membership functions. To improve the uncertainty handling of BK subproduct-based inference engines, an extension to Interval Type-2 Fuzzy
Sets (IT2FS) [15], [16] is needed. Thus, in this paper, our first contribution is to propose and extend the current $\mathrm{BK}$ subproduct to IT2FS.

Secondly, BK subproduct performs inferences by utilizing a set of common features that relate the inputs and outputs. In most cases, the BK subproduct treats all the features equally, i.e. the importance of all features are similar. However, in practical, not all these features have the same influences towards inference results, we argue that some features may have higher reliability or distinguishability than the others, and vice versa. Thus, our second contribution is to introduce weight parameter in the BK subproduct.

Work that similar to us in this case is [10], however, the implementation of the weight in this work required to fullfill a condition: $\sum_{i=1}^{N} w_{i}=1$ where $N$ is the number of features and $w$ is the weight of feature $i$. This condition is too restrictive for good implementation of weight because: (i) adding or decreasing features into consideration list will cause recalculation of all the weights. For instance, adding a new feature with weight $w_{N+1} \neq 0$ to existing feature list will cause the total weight become $\sum_{i=1}^{N+1} w_{i}$. It is easy to verify that $\sum_{i=1}^{N+1} w_{i}=1+w_{N+1}>1$ and the condition of total weight equal to 1 is not longer valid. Thus, a normalization is required so that the $\sum_{i=1}^{N+1} w_{i}=1$ is fulfilled. (ii) importance or influence of a feature is not intuitive - i.e. comparing a system with such condition to an implementation of weight where $w_{i} \in[0,1]$ for all $i$, the weights of the latter are much intuitive as weights close to 0 means less influence, while close to 1 means high influence. In [10], the weights can be small numbers that close to 0 even if they have high influence in the case of $N$ is a big number. Furthermore, this problem become much more complicated if new features are going to be added into consideration as one may not know what are the appropriate values that representing high (or low) influence. In contrast to [10], our weight term is $w_{i} \in[0,1]$ and condition: $\sum_{i=1}^{N} w_{i}=1$ is not required.

The rest of the paper are arranged as follow: In Section II, we provide a short revision on BK subproduct. Section III discusses the extension of BK subproduct from T1FS to IT2FS, along with a subsethood measure of IT2FS. In Section IV, we introduce the weight parameter to this newly developed IT2FS reasoning scheme. A case study is presented in Section $\mathrm{V}$ for demonstration purpose. Lastly, we conclude the paper 
in Section VI.

\section{BACKGROUND}

We start this section with a brief review on the definition of BK subproduct on crisp relations.

Assume that $A, B$ and $C$ are three crisp sets and $a, b$ and $c$ are general representation to the elements in the sets respectively. $R$ is defined as a relation from $A$ to $B$ such that $R \subseteq A \times B$; whereas $S$ is a relation from $B$ to $C$ such that $S \subseteq B \times C$. The converse relation of $S$ is denoted as $S^{T}$. The abbreviation $a R b$ shows that $a$ is in relation $R$ with $b$. Bandler and Kohout [4] defined (crisp) BK subproduct as follow:

Definition 1. BK subproduct is a composition of relations for $a$ and $c$ such that:

$$
R \triangleleft S=\{(a, c) \mid(a, c) \in A \times C \text { and } a R \subseteq S c\}
$$

where $a R=\{b \mid a R b\}$ is the image of $a$ after the projection of relation $R$ in the set $B$, while $S c=\{b \mid b S c\}$ is the image of $c$ after the projection of relation $S^{T}$ in the set $B$. BK subproduct is useful in retrieving relationships between elements of two indirectly associated sets, objects $A$ and targets $C$, if both sets can be associated with a set of common features, $B$.

Example 1 (Medical Inference Systems). Consider a set of $\mathrm{Pa}-$ tients $A$, a set of Diseases $C$, and a set of Signs/Symptoms $B$. $R$ is the relation from $A$ to $B$ indicating the signs/symptoms shown by patients, $S$ is a relation from $B$ to $C$, indicating the signs/symptoms used to characterize the diseases. Hence, BK subproduct $a^{\prime}(R \triangleleft S)$ provides all diseases that may show all signs/symptoms of patient $a^{\prime}$. In another word, all the signs/symptoms of patient $a^{\prime}$ are subset(s) of signs/symptoms of the disease(s) $c$.

It is trivial that Definition 1 is established on the subsethood of $a R$ in $S c$. In order to extend it to fuzzy relations, the fuzzy subsethood measure for T1FS is defined as:

Definition 2. For two T1FS, $P$ and $Q$ in the same universe $X$, the possibility of $P$ is a subset of $Q$ is given as follow:

$$
\pi(P \subseteq Q)=\bigwedge_{x \in X}\left(\mu_{P}(x) \rightarrow \mu_{Q}(x)\right)
$$

where $\rightarrow$ is fuzzy implication operators generally defined as "NOT $A$ OR $B$ " and $\wedge$ is the infimum operator. $\wedge$ can be considered as min function in harsh criterion or arithmetic mean in mean criterion [4].

Definition 3. With Eq. (1) and Eq. (2), Bandler and Kohout [4] defined the fuzzy BK subproduct as follow:

$$
R \triangleleft S(a, c)=\bigwedge_{b \in B}\left(R_{a b} \rightarrow S_{b c}\right)
$$

where $R_{a b}$ is the fuzzy degree to which $a R b$ is true and $S_{b c}$ is the fuzzy degree to which $b S c$ is true.

Later, De Baets and Kerre [17] discovered that the BK subproduct demonstrated a limitation in a specific condition and

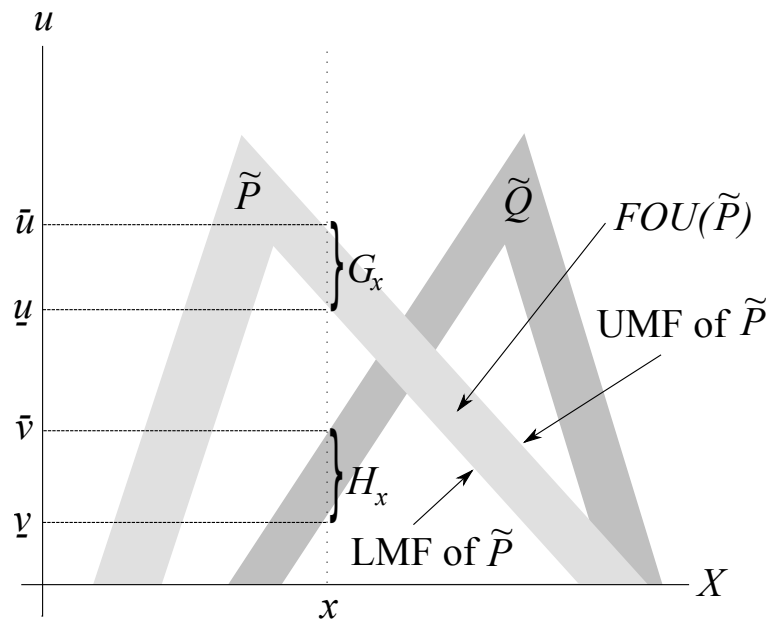

Fig. 1. Two IT2FS $\tilde{P}$ and $\tilde{Q}$ in the same universe $X$. The primary memberships for $\tilde{P}$ and $\tilde{Q}$ are denoted as $G_{x}$ and $H_{x}$ respectively. For $G_{x}$, $u$ denotes its secondary variable whereas $\underline{u}$ and $\bar{u}$ are its lower and upper limits respectively. The area in light gray is the Footprint of Uncertainty of $\tilde{P}$ and its lower and upper membership functions (LMF and UMF) are labeled in the figure.

improvement has been proposed by incorporating additional term. However, recent work [18] discovered that the additional term gives unwanted influence to the inference results. Thus, in this paper, the original version of fuzzy BK subproduct is employed as in [10].

\section{BK Subproduct With INTERVAL TyPe-2 FuZZY SETS}

\section{A. Interval Type-2 Fuzzy Sets}

An Interval Type-2 Fuzzy Set (IT2FS) is a special case of a Type-2 Fuzzy Set which the membership functions of an element is not point-value but an interval [15], [16].

An IT2FS denoted as $\tilde{P}$ in universe $X$ has elements $x \in \tilde{P}$. The membership functions of $x$ are intervals $G_{x}$. We also name these intervals as primary memberships. The lower and upper endpoints of a $G_{x}$ are given by an interval $[\underline{u}, \bar{u}]$ where $u$ are called secondary variables.

If we depict all the primary memberships of $x \in \tilde{P}$ in a $2 \mathrm{D}$ graph, the area covered by those primary memberships is the Footprint of Uncertainty (FOU) of $\tilde{P}$, denoted as $\operatorname{FOU}(\tilde{P})$. The outer margin of the FOU is the upper bound of FOU, called the Upper Membership Function (UMF), and the lower margin of the FOU is the lower bound of FOU, called the Lower Membership Function (LMF). An illustration is provided in Fig. 1.

\section{B. Subsethood Measure of IT2FS}

As fuzzy implication operators are only defined for point values, Eq. (2) needs to be extended so that the subsethood measurements for IT2FS can be computed. For this purpose, we incorporate the Representation Theorem [16].

Representation Theorem [16] suggests that an IT2FS can be represented as a collection of embedded T1FS. Assume that both axes $x$ and $u$ are discrete, an element $x_{i} \in \tilde{P}$ where 
$i=\{1,2, \cdots, I\}$ has $J_{i}$ discrete secondary variables in its FOU, namely $u_{i j}$ where $j=\left\{1,2, \cdots, J_{i}\right\}$. The number of embedded T1FS in $\tilde{P}$ is:

$$
\eta_{\tilde{P}}=\prod_{i=1}^{I} J_{i}
$$

Since these embedded T1FS are distributed evenly, the number of embedded T1FS pass through a secondary variable $u_{i j}$ is $\frac{\eta_{\tilde{P}}}{J_{i}}$.

In order to define the fuzzy subsethood measure, or the possibility $\tilde{P}$ is a subset of $\tilde{Q}$, i.e. $\pi(\tilde{P} \subseteq \tilde{Q})$, we use the notations of set $\tilde{Q}$ as shown in Fig. 1: the primary membership of $x \in \tilde{Q}$ is $H_{x}$ and secondary variable $v$, therefore $H_{x}=$ $[\underline{v}, \bar{v}]$. For an element $x_{i}, i=\{1,2, \cdots, I\}, K_{i}$ is the number of discrete variables $v_{i k}$ where $k=\left\{1,2, \cdots, K_{i}\right\}$. The total number of embedded T1FS in $\tilde{Q}$ is given by $\eta_{\tilde{Q}}=\prod_{i=1}^{I} K_{i}$ and number of embedded T1FS on a secondary variable $v_{i k}$ is $\frac{\eta_{\tilde{Q}}}{K_{i}}$.

To formulate the fuzzy subsethood measure of IT2FS, we start with evaluating an arbitrary pair of secondary variables $u_{i j}$ and $v_{i k}$ in $\tilde{P}$ and $\tilde{Q}$ respectively, on a same element $x_{i}$. The implication of these points to the subsethood measure $\pi(\tilde{P} \subseteq \tilde{Q})$ is $u_{i j} \rightarrow v_{i k}$. However, since there are $\frac{\eta_{\tilde{P}}}{J_{i}}$ of embedded T1FS on $u_{i j}$ and $\frac{\eta_{\tilde{Q}}}{K_{i}}$ on $v_{i k}$, this implication involves a number of $\frac{\eta_{\tilde{P}}}{J_{i}} \times \frac{\eta_{\tilde{Q}}}{K_{i}}$ pairs of embedded T1FS, thus it should be represented as:

$$
\frac{\eta_{\tilde{P}} \eta_{\tilde{Q}}}{J_{i} K_{i}}\left(u_{i j} \rightarrow v_{i k}\right)
$$

If $u_{i j}$ is the only discrete point in $G_{x}$, the subsethood measure can be evaluate by summing up the implication of this secondary variable to all the $v_{i j}$ (Fig 2):

$$
\frac{\eta_{\tilde{P}} \eta_{\tilde{Q}}}{J_{i} K_{i}} \sum_{k=1}^{K_{i}}\left(u_{i j} \rightarrow v_{i k}\right)
$$

In general cases, $G_{x}$ are intervals with more then one discrete points. Therefore, if we generalized Eq. (6) to all the $u_{i j}$ in the element and normalized it with the total number of embedded T1FS $\eta_{\tilde{P}} \eta_{\tilde{Q}}$, we have the fuzzy subsethood measure for the element $x_{i}$ :

$$
\pi(\tilde{P} \subseteq \tilde{Q})\left(x_{i}\right)=\frac{1}{J_{i} K_{i}} \sum_{j=1}^{J_{i}} \sum_{k=1}^{K_{i}}\left(u_{i j} \rightarrow v_{i k}\right)
$$

If we substitute Eq. (7) to Eq. (2) and the mean criterion is considered, the complete fuzzy subsethood measure for IT2FS is:

$$
\pi(\tilde{P} \subseteq \tilde{Q})=\frac{1}{I} \sum_{i=1}^{I} \frac{1}{J_{i} K_{i}} \sum_{j=1}^{J_{i}} \sum_{k=1}^{K_{i}}\left(u_{i j} \rightarrow v_{i k}\right)
$$

Employing a fuzzy implication operator, Eq. (8) will produce a subsethood measurement of $\tilde{P} \subseteq \tilde{Q}$ in interval $[0,1]$. However, we noticed that the subsethood measurements for crisp sets are Boolean (yes / no) and for T1FS are point-values. Therefore, we deduce that the fuzzy subsethood measurements for IT2FS should be intervals instead of point-values as of

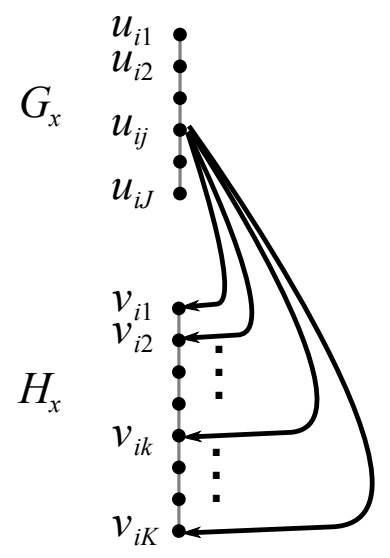

Fig. 2. Implication of a single discrete secondary variable in primary membership $G_{x}$ of $\tilde{P}$ to all the discretized secondary variables in primary membership $H_{x}$ of $\tilde{Q}$

[19]-[22]. Studies in [3], [23], [24] suggested that KleeneDienes and Łukasiewicz implication operators are among the two most suitable candidates for lower and upper bounds subsethood measurements. Thus, if $Y=[\underline{Y}, \bar{Y}]$ denotes lower and upper bounds of a subsethood measure:

$$
\begin{gathered}
\underline{Y}=\frac{1}{I} \sum_{i=1}^{I} \frac{1}{J_{i} K_{i}} \sum_{j=1}^{J_{i}} \sum_{k=1}^{K_{i}}\left(u_{i j} \rightarrow_{\mathrm{KD}} v_{i k}\right) \\
\bar{Y}=\frac{1}{I} \sum_{i=1}^{I} \frac{1}{J_{i} K_{i}} \sum_{j=1}^{J_{i}} \sum_{k=1}^{K_{i}}\left(u_{i j} \rightarrow_{£} v_{i k}\right)
\end{gathered}
$$

where $\rightarrow_{\mathrm{KD}}$ and $\rightarrow_{\mathrm{E}}$ are Kleene-Dienes and Łukasiewicz implication operators respectively:

$$
\begin{gathered}
\alpha \rightarrow_{\mathrm{KD}} \gamma=\max (\gamma, 1-\alpha) \\
\alpha \rightarrow_{£} \gamma=\min (1,1-\alpha+\gamma)
\end{gathered}
$$

\section{Extension of Fuzzy BK Subproduct}

Definition 1, 2 and 3 clearly showed that subsethood measure is the underlying idea of BK subproduct. Definition of BK subproduct is subsethood measure of $a R$ (image of an object $a$ in feature set $B$ under relation $R$ ) in another set $S c$ (image of a target $c$ in feature set $B$ under converse relation $S)$.

Thus, BK subproduct in term of IT2FS is ease to develop once we able to retrieve the subsethood measure of IT2FS. It is reasonable to assume that $\tilde{P}$ as $a \tilde{R}$ and $\tilde{Q}$ as $\tilde{S} c$. Due to the similarity between BK subproduct and subsethood measure, the notations used in the subsethood measure are used in BK subproduct as well. $I$ is the number of elements of $B . J_{i}$ is the interval that representing membership grade of $a \tilde{R} b_{i}$ and $u_{i j}$ are discretized values in the interval. Similarly, $K_{i}$ is the interval that representing membership grade of $b_{i} \tilde{S} c$ and $v_{i k}$ are discretized values in $K_{i}$ :

$$
\tilde{R} \triangleleft \tilde{S}(a, c)=\frac{1}{I} \sum_{i=1}^{I} \frac{1}{J_{i} K_{i}} \sum_{j=1}^{J_{i}} \sum_{k=1}^{K_{i}}\left(R_{a b_{i j}} \rightarrow S_{b_{i k} c}\right)
$$


Similar to Eq. (8), the results of Eq. (13) are intervals too. If the lower and upper bounds of these intervals are denoted as $\tilde{R} \unlhd \tilde{S}$ and $\tilde{R} \triangleleft \tilde{S}$ respectively, with Eq. (11) and Eq. (12), we obtain:

$$
\begin{aligned}
& \tilde{R} \unlhd \tilde{S}(a, c)=\frac{1}{I} \sum_{i=1}^{I} \frac{1}{J_{i} K_{i}} \sum_{j=1}^{J_{i}} \sum_{k=1}^{K_{i}} \max \left(S_{b_{i k} c}, 1-R_{a b_{i j}}\right) \\
& \tilde{R} \varangle \tilde{S}(a, c)=\frac{1}{I} \sum_{i=1}^{I} \frac{1}{J_{i} K_{i}} \sum_{j=1}^{J_{i}} \sum_{k=1}^{K_{i}} \min \left(1,1-R_{a b_{i j}}+S_{b_{i k} c}\right)
\end{aligned}
$$

\section{WeIght IN BK SubProduct BASEd INFERENCE ENGINE}

\section{A. Weight and BK Subproduct}

In most of the time, we can group criteria that we need to consider into a few criteria sets during reasoning. Among these criteria sets, some of them might have higher influence over the others in a decision making process. For instance, let us look back to Example 1. A physician may consider the following 4 criteria sets during a medical diagnosis, namely symptoms, patient personal history, family history and environmental issues. However, not all the criteria sets having the same influence in the medical diagnosis. In the diagnosis of diseases such as breast cancer, physicians may take more consideration on symptoms (higher influence) found on patients compare to environmental issues (low influence). Hence, weights are meaningful in representing the influence of the criteria sets.

However, one should note that the weight should not be confused with the strength of criteria in the criteria set. Similar case is applied to inference engines based on BK subproduct. Instead of criteria, features are considered here. Membership functions of objects-features relations $(\tilde{R})$ and features-target relations $(\tilde{S})$ are the "strength of criteria" that determine the results of inferencing. While features forming feature sets, influence of each feature sets are represented as weight. Weight applies to each feature set and we model the weight with IT2FS in this paper.

As mentioned earlier, subsethood measure is the fundamental of fuzzy BK subproduct. Thus, one might argue that it is inappropriate to implement weight in BK subproduct based inference engines because there is no well defined weighted subsethood measure in the literature. In fact, the weights are applied to the feature sets rather then the subsethood measurements. We explain the argument with a multiple feature sets model explained below.

Assume that the features in set $B$ can be grouped into multiple feature sets $B_{m}, m=\{1,2, \cdots, M\}$. Each feature set has a number of features. The relation between $A$ and $B_{m}$ is $\tilde{R}_{m}$, whereas $\tilde{S}_{m}$ is the relation between $B_{m}$ and $C$. In this case, the images of $a \tilde{R}_{m}$ and $\tilde{S}_{m} c$ are $\tilde{P}_{m}$ and $\tilde{Q}_{m}$ respectively. Subsethood measure of $\tilde{P}_{m} \subseteq \tilde{Q}_{m}$ gives $\tilde{R}_{m} \triangleleft \tilde{S}_{m}(a, c)$ (Fig. 3).

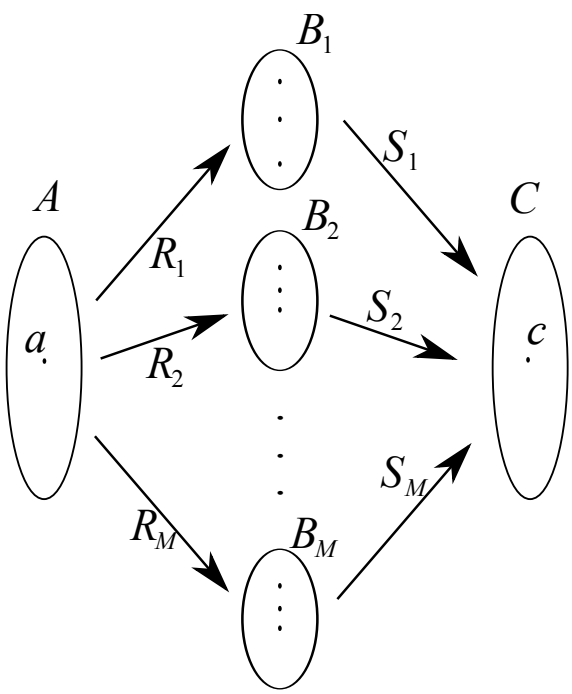

Fig. 3. Breaking up set $B$ to multiple feature sets to form weighted BK subproduct.

Each feature set carries different weight. Assume that the weight of $\tilde{R}_{m} \triangleleft \tilde{S}_{m}(a, c)$ is $\tilde{W}_{m}$, the normalized aggregation of all the composition of relations is given as:

$$
\tilde{R} \triangleleft \tilde{S}(a, c)=\frac{\sum_{m=1}^{M} \tilde{W}_{m}\left(\tilde{R}_{m} \triangleleft \tilde{S}_{m}(a, c)\right)}{\sum_{m=1}^{M} \tilde{W}_{m}}
$$

Eq. (16) gives the weighted measure of BK subproduct. Here, since all $\tilde{R}_{m} \triangleleft \tilde{S}_{m}(a, c)$ are intervals that only exist as numerators, whereas $\tilde{W}_{m}$ are IT2FS, the results of computations based on Eq. (16) are always IT2FS. We show the details of solving Eq. (16) in the following subsection.

\section{B. Solving the Weighted Average}

Solving Eq. (16) is easy if all the parameters are crisp numbers. However, these parameters are fuzzy, and so the solution become slightly complicated, especially with a term $1 / \sum_{m=1}^{M} \tilde{W}_{m}$. One of the closest problem as us that solved in the literature is Fuzzy Weighted Average (FWA) [25]. FWA solved the problems in the form of:

$$
f=\sum_{m=1}^{M} \omega_{m} \chi_{m} / \sum_{m=1}^{M} \omega_{m}
$$

where all $\chi_{m}$ and $\omega_{m}$ are T1FS. Wu and Mendel [26], [27] extended FWA to form Linguistic Weighted Average (LWA), which solve the problem where all $\chi_{m}$ and $\omega_{m}$ are IT2FS. Both FWA and LWA uses $\alpha$-cut decomposition theorem [28] in solving the problems. With $\alpha$-cut decomposition theorem, instead of performing calculation directly on the sets $\left(\chi_{m}\right.$ and $\left.\omega_{m}\right)$ as whole, a number of $(\delta-1) \alpha$-cuts are taken to break the sets into $\delta$ intervals. For each interval $\alpha_{\iota}, 1 \leq \iota \leq \delta$, perform calculation that suppose to for the sets to yield an 
TABLE I

THIS TABLE SHOWS $R_{a b}$ AND $S_{b c}$, THE RELATIONS BETWEEN THE THREE PATIENTS IN SET $A$, ELEVEN FEATURES IN SET $B$ AND A DISEASE IN SET $C$.

\begin{tabular}{c|ccc|c}
\hline$R_{a b}$ or $S_{b c}$ & $a_{1}$ & $a_{2}$ & $a_{3}$ & $c_{1}$ \\
\hline$b_{11}$ & {$[0.1,0.3]$} & {$[0.9,1.0]$} & {$[0.4,0.5]$} & {$[0.2,0.3]$} \\
$b_{12}$ & {$[0.2,0.3]$} & {$[0.8,0.9]$} & {$[0.7,0.8]$} & {$[0.1,0.2]$} \\
$b_{13}$ & {$[0.3,0.5]$} & {$[0.8,1.0]$} & {$[0.6,0.9]$} & {$[0.4,0.4]$} \\
$b_{14}$ & {$[0.7,0.8]$} & {$[0.8,1.0]$} & {$[0.8,0.9]$} & {$[0.7,0.8]$} \\
$b_{21}$ & {$[0.9,1.0]$} & {$[0.2,0.2]$} & {$[0.7,0.8]$} & {$[0.2,0.3]$} \\
$b_{22}$ & {$[0.6,0.7]$} & {$[0.6,0.7]$} & {$[0.7,0.8]$} & {$[0.4,0.6]$} \\
$b_{31}$ & {$[0.7,0.9]$} & {$[0.1,0.3]$} & {$[0.7,0.8]$} & {$[0.1,0.2]$} \\
$b_{32}$ & {$[0.8,0.9]$} & {$[0.1,0.2]$} & {$[0.7,0.8]$} & {$[0.1,0.2]$} \\
$b_{33}$ & {$[0.6,0.7]$} & {$[0.2,0.5]$} & {$[0.1,0.1]$} & {$[0.3,0.4]$} \\
$b_{41}$ & {$[0.9,1.0]$} & {$[0.2,0.2]$} & {$[0.0,0.1]$} & {$[0.1,0.2]$} \\
$b_{42}$ & {$[0.8,0.9]$} & {$[0.5,0.8]$} & {$[0.1,0.1]$} & {$[0.7,0.8]$} \\
\hline
\end{tabular}

interval $f_{\iota}$. The composition of all the $f_{\iota}$ with corresponding $\alpha$-cuts will form the corresponding set $f$.

With the existing findings, Eq. (16) can be solved easily. We adopted the solution of LWA in [26], [27] by assuming that the intervals $\tilde{R}_{m} \triangleleft \tilde{S}_{m}(a, c)$ in Eq. (16) are special cases of IT2FS, where these fuzzy sets have rectangle membership functions and the $\operatorname{UMF}\left(\tilde{R}_{m} \triangleleft \tilde{S}_{m}(a, c)\right)=\operatorname{LMF}\left(\tilde{R}_{m} \triangleleft \tilde{S}_{m}(a, c)\right)$. From here onwards, we denote $\tilde{R}_{m} \triangleleft \tilde{S}_{m}(a, c)$ as $Z_{m}$ and the lower and upper bounds of $Z_{m}$ are denoted as $\underline{Z}_{m}$ and $\bar{Z}_{m}$ respectively. Thus, follow this notation scheme, $\tilde{R} \triangleleft \tilde{S}(a, c)$ is denoted as $\tilde{Z}$.

Since the FOU of $\tilde{Z}$ is determined by $\operatorname{UMF}(\tilde{Z})$ and $\operatorname{LMF}(\tilde{Z})$, we can find $\tilde{Z}$ by calculating these two boundaries only. In [27], Wu and Mendel proved that the height of the output sets from LWA are equal to the minimum height of all $Z_{m}$ and $W_{m}$. In our case, since all $\operatorname{UMF}\left(\tilde{W}_{m}\right)$ are normal and $Z_{m}$ are intervals, the height of an $\operatorname{UMF}(\tilde{Z})$ is unity. On the other hand, the height of a $\operatorname{LMF}(\tilde{Z})$ is totally depends on $\operatorname{LMF}\left(\tilde{W}_{m}\right)$. Assume that all the $\tilde{W}_{m}$ are having trapezoidal (or triangular) shape FOU, the shape of $\tilde{Z}$ should be trapezoidal (or triangular) as well (Fig. 4).

As described earlier in this section, the solution of Eq. (16) starts with taking $(\delta-1) \alpha$-cuts to yield $\delta$ intervals for each set. Thus, the rest of the work is simplified to find the intervals that represent the FOU of $\tilde{Z}$ corresponding to each $\alpha$-cut. For this purpose, we refer to the notations that described in Fig. 4.

By referring to the results of LWA [26], [27], for each $\alpha$ cut, the corresponding boundaries of $\operatorname{UMF}(\tilde{Z})$ and $\operatorname{LMF}(\tilde{Z})$ can be obtained by sorting $\bar{Z}_{m}$ and $\underline{Z}_{m}$ first, then substituting the corresponding values into the following equations:

$$
\begin{aligned}
& z_{1}=\frac{\sum_{m=1}^{\beta_{1}} w_{m 4} \underline{Z}_{m}+\sum_{m=\beta_{1}+1}^{M} w_{m 1} \underline{Z}_{m}}{\sum_{m=1}^{\beta_{1}} w_{m 4}+\sum_{m=\beta_{1}+1}^{M} w_{m 1}} \\
& z_{2}=\frac{\sum_{m=1}^{\beta_{2}} w_{m 3} \underline{Z}_{m}+\sum_{m=\beta_{2}+1}^{M} w_{m 2} \underline{Z}_{m}}{\sum_{m=1}^{\beta_{2}} w_{m 3}+\sum_{m=\beta_{2}+1}^{M} w_{m 2}} \\
& z_{3}=\frac{\sum_{m=1}^{\beta_{3}} w_{m 2} \bar{Z}_{m}+\sum_{m=\beta_{3}+1}^{M} w_{m 3} \bar{Z}_{m}}{\sum_{m=1}^{\beta_{3}} w_{m 2}+\sum_{m=\beta_{3}+1}^{M} w_{m 3}}
\end{aligned}
$$

TABLE II

INFERENCE RESULTS IF THE WEIGHTS ARE NOT CONSIDERED.

\begin{tabular}{c|ccc}
\hline & $a_{1}$ & $a_{2}$ & $a_{3}$ \\
\hline$c_{1}$ & {$[0.48,0.68]$} & {$[0.61,0.78]$} & {$[0.54,0.71]$} \\
\hline$z_{4}=\frac{\sum_{m=1}^{\beta_{4}} w_{m 1} \bar{Z}_{m}+\sum_{m=\beta_{4}+1}^{M} w_{m 4} \bar{Z}_{m}}{\sum_{m=1}^{\beta_{4}} w_{m 1}+\sum_{m=\beta_{4}+1}^{M} w_{m 4}}$
\end{tabular}

In these equations, $\beta_{1}, \beta_{2}, \beta_{3}$ and $\beta_{4}$ are the switching points in the range $[1, M]$ calculated with Karnik-Mendel algorithm [29], [30] such that:

$$
\begin{aligned}
& \underline{Z}_{\beta_{1}} \leq z_{1} \leq \underline{Z}_{\beta_{1}+1} \\
& \underline{Z}_{\beta_{2}} \leq z_{2} \leq \underline{Z}_{\beta_{2}+1} \\
& \bar{Z}_{\beta_{3}} \leq z_{3} \leq \bar{Z}_{\beta_{3}+1} \\
& \bar{Z}_{\beta_{4}} \leq z_{4} \leq \bar{Z}_{\beta_{4}+1}
\end{aligned}
$$

\section{Results Interpretation}

Compositions of the results obtained from Eq. (18)-(21) give a set of IT2FS, and the meaning carried by this set of IT2FS is application dependent. In some applications, ranking algorithms suggested in [31], [32] are useful if comparisons between these IT2FS are needed to find the highest order one. Engineering control applications might fall to this category. In some other cases, the results can be compared with a set of predefined IT2FS using similarity measure [19], [32].

\section{CAse Study}

In this section, we extend Example 1 to a case study. The purpose of this is to demonstrate the computation procedure and the influence of weights in inferencing.

Consider $A=\left\{a_{1}, a_{2}, a_{3}\right\}$ as a set of three patients and $C=\left\{c_{1}\right\}$ as a set with 1 disease. $B$ is a set of 11 features that can be used in this medical diagnosis. This features are grouped into 4 sub feature sets (e.g: sign/symptoms, patient history, family history, environmental issues) such that : $B_{1}=$ $\left\{b_{11}, b_{12}, b_{13}, b_{14}\right\}, B_{2}=\left\{b_{21}, b_{22}\right\}, B_{3}=\left\{b_{31}, b_{32}, b_{33}\right\}$ and $B_{4}=\left\{b_{41}, b_{42}\right\}$. Table I shows the relations between $A, B$ and $C$. The weights of $B_{1}, B_{2}, B_{3}$ and $B_{4}$ are $W_{1}, W_{2}, W_{3}$ and $W_{4}$ respectively. Refer to Fig. 5 and Table IV for details of these weights.

\section{A. Results}

If weights are not considered in this case, grouping all features into multiple feature sets is meaningless. Thus, just consider that $B$ is a set with all the 11 features, applying Eq. (14) and Eq. (15) give the results presented in Table II.

To consider the weights, firstly we compute the inference results for each group of features using Eq. (14) and Eq. (15). The results are presented in Table III.

As described in Section IV-B, solving this weighted BK subproduct based inferencing need multiple $\alpha$-cuts. However, since all the membership functions in this case are trapezoidal, three $\alpha$ or strong $\alpha$-cuts is effective to compute the answer, i.e. $\alpha=0+, 0.6$ and 1 . 


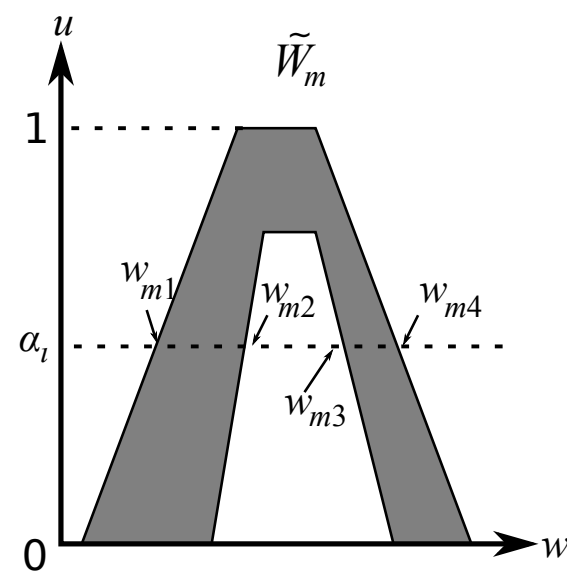

(i)

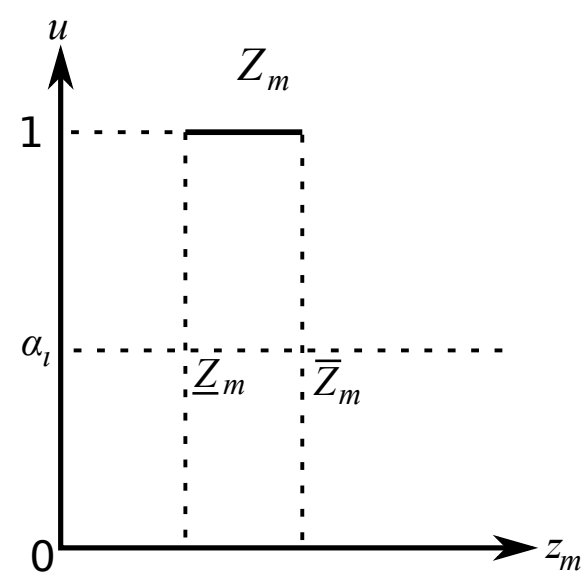

(ii)

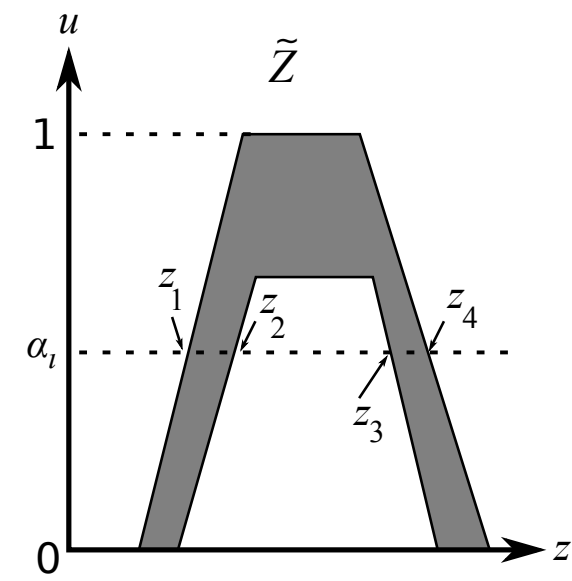

(iii)

Fig. 4. Shape and notations of sets. (i) $\tilde{W}_{m}$ : for an $\alpha$-cut $\alpha_{\iota}, w_{\iota m 1}$ and $w_{\iota m 4}$ should be the leftmost and rightmost values of UMF( $\left.\tilde{W}_{m}\right)$ respectively at $\alpha_{\iota}$. However, we leave out the variable $\iota$ as an subscript of all variables here and follows because it is independent from the calculation of each iteration, and to make the equations look more concise. Therefore, these variables become $w_{m 1}$ and $w_{m 4}$. Similarly, $w_{m 2}$ and $w_{m 3}$ are the leftmost and rightmost values of $\operatorname{LMF}\left(\tilde{W}_{m}\right)$ respectively. (ii) $Z_{m}: \underline{Z}_{m}$ is the lower bound of interval $Z_{m}$, whereas $\bar{Z}_{m}$ is the upper bound of this interval. (iii) $\tilde{Z}$ : for an $\alpha$-cut $\alpha_{\iota}, z_{1}$ and $z_{4}$ are the leftmost and rightmost values of $\operatorname{UMF}(\tilde{Z})$ respectively. Similarly, $z_{2}$ and $z_{3}$ are the leftmost and rightmost values of LMF $(\tilde{Z})$ respectively.

TABLE III

INFERENCE RESULTS OF EACH GROUP OF FEATURES FOR EACH PATIENT.

\begin{tabular}{c|cccc}
\hline & $Z_{1}$ & $Z_{2}$ & $Z_{3}$ & $Z_{4}$ \\
\hline$a_{1}$ & {$[0.73,0.96]$} & {$[0.38,0.58]$} & {$[0.26,0.45]$} & {$[0.45,0.55]$} \\
$a_{2}$ & {$[0.39,0.49]$} & {$[0.65,0.93]$} & {$[0.77,0.95]$} & {$[0.78,0.97]$} \\
$a_{3}$ & {$[0.49,0.69]$} & {$[0.39,0.63]$} & {$[0.47,0.60]$} & {$[0.93,1.00]$} \\
\hline
\end{tabular}

TABLE IV

THIS TABLE PRESENTS THE NUMERICAL VALUES OF THE PARAMETERS THAT FORMED WEIGHTS IN FIG. 5.

\begin{tabular}{l||c|cccccccc}
\hline & $u^{\prime}$ & $w_{1}^{\prime}$ & $w_{2}^{\prime}$ & $w_{3}^{\prime}$ & $w_{4}^{\prime}$ & $w_{5}^{\prime}$ & $w_{6}^{\prime}$ & $w_{7}^{\prime}$ & $w_{8}^{\prime}$ \\
\hline$W_{1}$ & 0.70 & 0.70 & 0.80 & 0.85 & 0.95 & 1.00 & 1.00 & 1.00 & 1.00 \\
$W_{2}$ & 0.60 & 0.10 & 0.15 & 0.20 & 0.25 & 0.30 & 0.35 & 0.40 & 0.45 \\
$W_{3}$ & 0.70 & 0.00 & 0.00 & 0.00 & 0.00 & 0.00 & 0.10 & 0.15 & 0.25 \\
$W_{4}$ & 0.80 & 0.00 & 0.10 & 0.20 & 0.20 & 0.20 & 0.25 & 0.35 & 0.40 \\
\hline
\end{tabular}

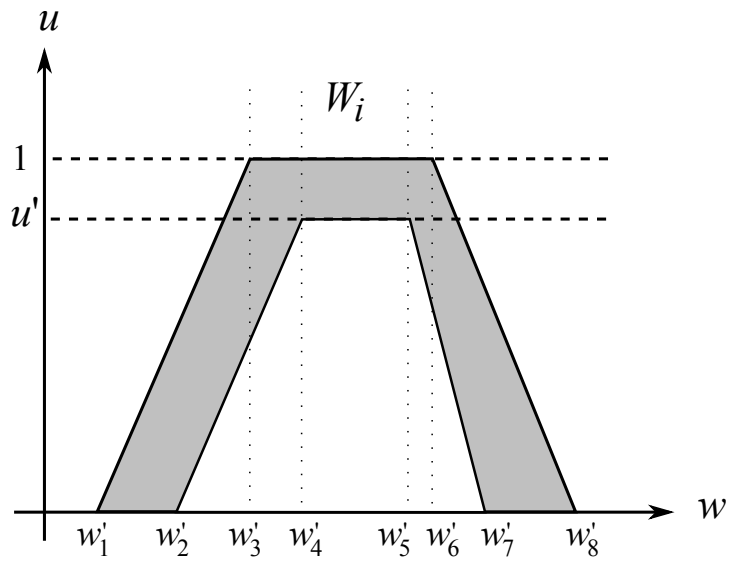

Fig. 5. A weight and its parameters modeled as IT2FS with trapezoidal membership function.

With Eq. (18) and Eq. (21), $\operatorname{UMF}(\tilde{Z})$ can be computed, whereas Eq. (19) and Eq. (20) give the results of $\operatorname{LMF}(\tilde{Z})$. These results are presented in Fig. 6. To make the comparison with Table II easier, interval results in Table II are presented as a "black bar" in Fig. 6.

\section{B. Discussion}

In Fig. 6(a), although defuzzification is needed so that the weighted inference result (represented with IT2FS) can be compared with its unweighted result (represented with interval), but it is trivial that the weighted inference result gives stronger suggestion that the patient $a_{1}$ is suffering with disease $c_{1}$.

On the other hand, Fig. 6(b) shows a different scenario. Compare to the result of weighted inference, the unweighted result gives stronger suggestion that patient $a_{2}$ is suffering with the illness. The cases of these two patients, $a_{1}$ and $a_{2}$, clearly showed that the influence of weight parameter in inferencing.

For the case of patient $a_{3}$, we can observe the following: (i) with the influence of weight, both the results have higher coherency compare to the cases of $a_{1}$ and $a_{2}$; (ii) although its unweighted result is quite similar to the unweighted result of of $a_{1}$, but their weighted results give different impression. Comparatively, its weighted result is more similar to the weighted result of patient $a_{2}$.

Overall, considering weight parameter in BK subproduct based inferencing gives direct impact in the inference results. This impact can be in any direction. Although there is no ground truth to compare with in this simulated case study, the results with weights considered are expected to be more reliable since more details, including the significance of each feature is considered.

\section{CONCLUSION}

Studies such as [1], [2], [33] have proved that BK subproduct has its advantages in formulating reasoning schemes. However, this does not means that the schemes developed from BK subproduct have no limitations. In this paper, we 


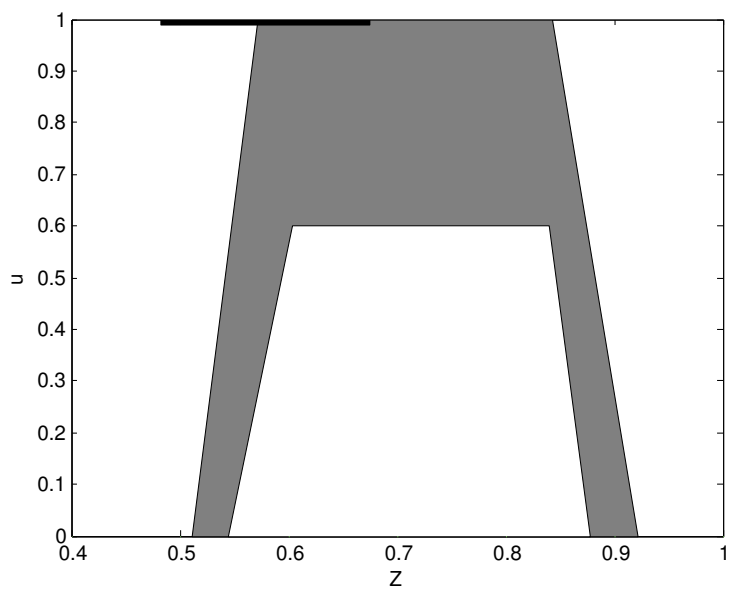

(a) Inference result for patient $a_{1}$

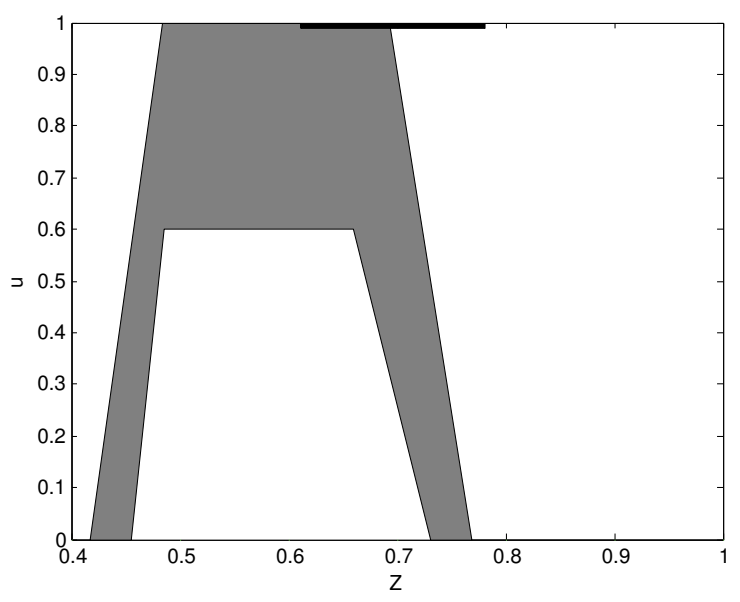

(b) Inference result for patient $a_{2}$

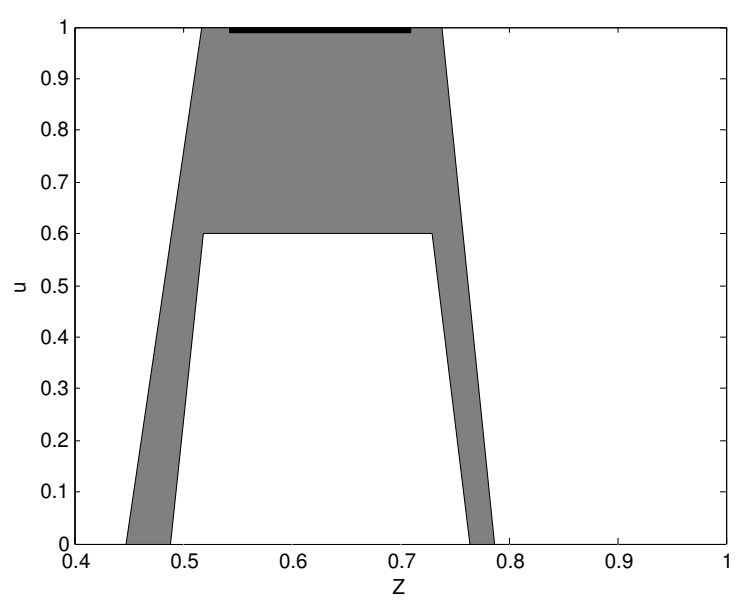

(c) Inference result for patient $a_{3}$

Fig. 6. Final results of weighted inference are presented in term of trapezoidal membership functions. For comparisons, the results if grouping and weights are not considered (Table II) are presented as black bar in corresponding graphs. first propose two enhancements on BK subproduct. These enhancements are: (i) interval type-2 fuzzy sets are used instead of ordinary type-1 fuzzy sets. This enhancement allows BK subproduct based inference engines to have better capability in handling uncertainties; and (ii) weight parameter is added to form weighted interval type-2 fuzzy based inference scheme. This enhancement allows the inference schemes to consider multiple groups of features at the same time, even though they have different levels of influence in the inference.

Due to BK subproduct is based on fuzzy subsethood measure, we also extended the implication operators based subsethood measure [4] to work with IT2FS. The outcome, however, does not have straight forward solution because the terms $\tilde{W}_{m}$ are IT2FS while $\tilde{R}_{m} \triangleleft \tilde{S}_{m}(a, c)$ are intervals. We adopted the solution of LWA [26], [27] by assuming that the intervals $\tilde{R}_{m} \triangleleft \tilde{S}_{m}(a, c)$ are special cases of IT2FS. Thus, the outcome can be solved easily by fining the FOU of $\tilde{R} \triangleleft \tilde{S}(a, c)$.

A simulated case study is provided to demonstrate the computing procedure of this weighted BK subproduct based inference engine. Our future work is looking forward to apply this into human motion analysis [34]-[36] and complex scene understanding context [37].

\section{REFERENCES}

[1] U. Bodenhofer, M. Dankova, M. Stepnicka, and V. Novak, "A Plea for the Usefulness of the Deductive Interpretation of Fuzzy Rules in Engineering Applications," 2007 IEEE International Fuzzy Systems Conference, pp. 1-6, Jun. 2007.

[2] M. Stepnicka and B. Jayaram, "On the Suitability of the BandlerKohout Subproduct as an Inference Mechanism," Fuzzy Systems, IEEE Transactions on, vol. 18, no. 2, pp. 285-298, 2010.

[3] L. J. Kohout and W. Bandler, "Semantics of implication operators and fuzzy relational products," International Journal of Man-Machine Studies, vol. 12, no. 1, pp. 89-116, Jan. 1980.

[4] — , "Fuzzy Power Sets And Fuzzy Implication Operators," Fuzzy Sets and Systems, vol. 4, pp. 13-30, 1980.

[5] L. Zadeh, "Outline of a new approach to the analysis of complex systems and decision processes," Systems, Man and Cybernetics, IEEE Transactions on, no. 1, pp. 28-44, 1973.

[6] L. J. Kohout, I. Stabile, H. Kalantar, and F. San-Andres, "Parallel intervalbased reasoning in medical knowledge-based system Clinaid," Reliable Computing, vol. 1, no. 2, pp. 109-140, 1995.

[7] K. M. Yew and L. J. Kohout, "Interval-based reasoning in medical diagnosis," in Proceedings Intelligent Information Systems. IIS'97. IEEE Comput. Soc, 1997, pp. 32-36.

[8] L.-d. Bui and Y.-g. Kim, "An Obstacle-avoidance Technique for Autonomous Underwater Vehicles Based On BK-products of Fuzzy Relation," Fuzzy Sets and Systems, vol. 157, pp. 560 - 577, 2006.

[9] L. J. Kohout and W. Bandler, "Relational-Product Architectures for Information Processing," Information Sciences, vol. 37, pp. 25-37, 1985.

[10] R. Groenemans, V. E. Ranst, and E. Kerre, "Fuzzy relational calculus in land evaluation," Geoderma, vol. 77, pp. 283-298, 1997.

[11] L. J. Kohout, "Power Sets, Implications and Set Inclusions Revisited Retrospect and Prospect: A Review of Bandler and Kohouts Paper and a Survey of 30 Years of Subsequent Developments," in Views on Fuzzy Sets and Systems from Different Perspectives. Springer, 2009, vol. 243, pp. 129-159.

[12] E. Vats, C. K. Lim, and C. S. Chan, "A BK subproduct approach for scene classification," in Proceedings of the IIEEJ Image Electronics and Visual Computing Workshop, Kuching, Malaysia, 2012.

[13] J. Mendel, "Interval type-2 fuzzy logic systems: theory and design," IEEE Transactions on Fuzzy Systems, vol. 8, no. 5, pp. 535-550, 2000.

[14] — "Type-2 fuzzy sets: some questions and answers," IEEE Neural Networks Society, no. August, pp. 10-13, 2003. 
[15] L. A. Zadeh, "The concept of a linguistic variable and its application to approximate reasoning-I," Information Sciences, vol. 8, no. 3, pp. 199$249,1975$.

[16] J. M. Mendel and R. John, "Type-2 fuzzy sets made simple," IEEE Transactions on Fuzzy Systems, vol. 10, no. 2, p. 117-127, Apr. 2002.

[17] B. De Baets and E. Kerre, "Fuzzy relational compositions," Fuzzy Sets and Systems, vol. 60, no. 1, pp. 109-120, Nov. 1993.

[18] C. K. Lim and C. S. Chan, "Logical Connectives and Operativeness Of BK Subproduct In Fuzzy Inferencing," International Journal of Fuzzy Systems, vol. 13, no. 4, pp. 237-245, 2011.

[19] H. Nguyen and V. Kreinovich, "Computing Degrees of Subsethood and Similarity for Interval-Valued Fuzzy Sets : Fast Algorithms," in Proceedings of the 9th International Conference on Intelligent Technologies. Samui, Thailand: Citeseer, 2008, pp. 47-55.

[20] M.-S. Yang and D.-C. Lin, "On similarity and inclusion measures between type-2 fuzzy sets with an application to clustering," Computers \& Mathematics with Applications, vol. 57, no. 6, pp. 896-907, Mar. 2009.

[21] G. Zheng, J. Xiao, Y. Zhang, and G. Shi, "An inclusion measure between general type-2 fuzzy sets," in Fuzzy Systems and Knowledge Discovery (FSKD), 2010 Seventh International Conference on, vol. 1, no. Fskd. IEEE, 2010, pp. 99-103.

[22] J. T. Rickard, J. Aisbett, and G. Greb, "Fuzzy Subsethood for Fuzzy Sets of Type-2 and Generalized Type-n," IEEE Transactions on Fuzzy Systems, vol. 17, no. 1, pp. 50-60, Feb. 2009.

[23] L. J. Kohout and W. Bandler, "How the checklist paradigm elucidates the semantics of fuzzy inference," IEEE International Conference on Fuzzy Systems, pp. 571-578, 1992.

[24] C. K. Lim and C. S. Chan, "Fuzzy set and multi descriptions property," in 2012 IEEE International Conference on Fuzzy Systems, IEEE, pp. 1-8, Jun. 2012.

[25] W. M. Dong and F. S. Wong, "Fuzzy weighted averages and implementation of the extension principle," Fuzzy Sets and Systems, vol. 21, pp. 183-199, 1987.
[26] D. Wu and J. Mendel, "Aggregation using the linguistic weighted average and interval type-2 fuzzy sets," Fuzzy Systems, IEEE Transactions on, vol. 15, no. 6, pp. 1145-1161, 2007.

[27] — "Corrections to Aggregation Using the Linguistic Weighted Average and Interval Type-2 Fuzzy Sets,' Fuzzy Systems, IEEE Transactions on, vol. 16, no. 6, pp. 1664-1666, 2008.

[28] G. J. Klir and B. Yuan, Fuzzy Sets And Fuzzy Logic: Theory And Applications. New Jersey: Prentice Hall, 1995.

[29] F. Liu and J. Mendel, "Aggregation Using the Fuzzy Weighted Average as Computed by the KarnikMendel Algorithms," IEEE Transactions on Fuzzy Systems, vol. 16, no. 1, pp. 1-12, 2008.

[30] J. Mendel, "Enhanced Karnik-Mendel Algorithms," IEEE Transactions on Fuzzy Systems, vol. 17, no. 4, pp. 923-934, Aug. 2009.

[31] H. B. Mitchell, "Ranking type-2 fuzzy numbers," Fuzzy Systems, IEEE Transactions on, vol. 14, no. 2, pp. 287-294, 2006.

[32] D. Wu and J. M. Mendel, "A comparative study of ranking methods, similarity measures and uncertainty measures for interval type-2 fuzzy sets," Information Sciences, vol. 179, no. 8, pp. 1169-1192, Mar. 2009.

[33] E. Kerre, "An overview of fuzzy relational calculus and its applications," in MDAI 07. Springer, 2007, pp. 1-13.

[34] C. S. Chan and H. Liu, "Fuzzy qualitative human motion analysis," IEEE T. Fuzzy Systems, vol. 17, no. 4, pp. 851-862, 2009.

[35] C. S. Chan, H. Liu, D. J. Brown, and N. Kubota, "A fuzzy qualitative approach to human motion recognition," in FUZZ-IEEE 2008, IEEE International Conference on Fuzzy Systems, Hong Kong, China, 1-6 June, 2008, Proceedings, 2008, pp. 1242-1249.

[36] C. S. Chan, H. Liu, and D. J. Brown, "Recognition of human motion from qualitative normalised templates," Journal of Intelligent and Robotic Systems, vol. 48, no. 1, pp. 79-95, 2007.

[37] C. H. Lim and C. S. Chan, "A fuzzy qualitative approach for scene classification," in FUZZ-IEEE 2012, IEEE International Conference on Fuzzy Systems, Brisbane, Australia, June 10-15, 2012, Proceedings, 2012, pp. 1-8. 